\title{
Concepts for Pilot Interaction with an Automated NOE Obstacle-Avoidance System
}

R. A. Coppenbarger and V. H. L. Cheng

(NASA-TM-103960) CONCEPTS FOR

PILOT INTERACTION WITH AN AUTOMATED

NOE OBSTACLE-AVOIDANCE SYSTEM

(NASA) $16 \mathrm{P}$
N93-19105

Unclas

$63 / 08 \quad 0146083$

December 1992 


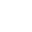

. 
NASA Technical Memorandum 103960

\section{Concepts for Pilot Interaction with an Automated NOE Obstacle-Avoidance System}

R. A. Coppenbarger and V. H. L. Cheng, Ames Research Center, Moffett Field, California

December 1992

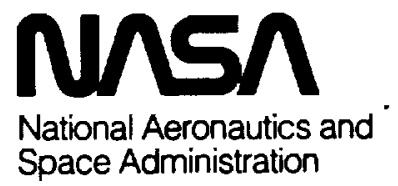


. 


$\begin{array}{ll}\text { Nomenclature } & \text { acceleration } \\ a & \text { automatic } \\ c & \text { command } \\ g & \text { acceleration due to gravity } \\ \dot{h}_{c l} & \text { terrain clearance altitude rate } \\ k_{r}, k_{v} & \text { control-law constants } \\ R & \text { radius of curvature } \\ r & \text { reference point } \\ \Delta r_{H} & \text { horizontal position vector } \\ r e f & \text { reference } \\ T_{I B} & \text { body-to-inertial transformation }\end{array}$

iw thumb-wheel

$V_{H} \quad$ horizontal velocity

$V_{d} \quad$ desired nominal ground speed

$V_{2} \quad$ vertical velocity

$\delta_{\text {lat }}$ roll cyclic

$\delta_{\text {Ion pitch cyclic }}$

$\gamma \quad$ flight-path angle

$\eta \quad$ PDG turn-magnitude angle

$\tau$ control-law time constant

$\Psi \quad$ heading angle

$\phi$ bank attitude angle 


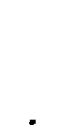




\section{Summary}

The problem of providing an appropriate method by which a human pilot interacts with an automated nap-of-the-Earth rotorcraft guidance and control system is addressed. This problem is closely related to the broader question of what level and degree of automation is effective at reducing pilot workload during low-altitude flight missions requiring obstacle avoidance. A systematic approach for establishing the possible combinations of manual versus automatic authority over relevant guidance and control functions is first presented. From these possibilities, three candidate concepts are selected; selection is based upon their potential for practical implementation and reduction in pilot workload. This paper describes the selection of these three pilot-interaction concepts and the mathematical models for their implementation.

\section{Introduction}

Pilots flying rotorcraft close to the ground in nap-of-theEarth (NOE) flight are confronted with unique guidance and control tasks that require a high degree of skill and concentration. These flight tasks, which can be intensified by low-visibility and/or battlefield conditions, include longrange mission planning as well as real-time pilotage involving obstacle avoidance (ref. 1). As part of an ongoing effort at Ames Research Center to reduce pilot workload by automating tasks for NOE flight, a fully automated NOE guidance and control system has been developed and implemented in a real-time simulation. The technical emphasis of this system has been on the development of guidance laws that select open paths for safe maneuvering based upon the identification of obstacles from onboard sensor information (refs. 2-5).

With the guidance and control functions developed for autonomous flight, the next step towards an operational piloted system is to develop an intelligent means by which a human pilot can interact with the automated subsystems. In developing a suitable pilot interface, the level and degree of automation useful to a pilot in performing his flight tasks must be determined. In this paper the degree of automation and the pilot interface are discussed within the context of military NOE missions. Automation in this flight regime is motivated by the desire to reduce pilot workload without compromising pilot confidence and safety. It is apparent that the level of automation and associated pilot interface are strongly related to pilot acceptability, which is crucial to the success of a practical rotorcraft system.

The issue of pilot acceptability of automated NOE technology was investigated by Systems Technology, Inc. (STI), under a recent NASA contract (ref. 6). This research was an initial investigation of the many aspects of partially and fully automated NOE flight. The concerns of this study included obstacle-avoidance maneuvers, pilot displays, pilot workload, and handling qualities. The simulation was carried out on the Vertical Motion Simulator (VMS) at Ames Research Center and involved four NASA test pilots. Qualitative results from pilot discussions and ratings emphasized pilot interaction as being a crucial problem in need of further research.

The purpose of this paper is to provide conceptual configurations and descriptions of an automated NOE sysiem in synergism with a human pilot. The existing Ames fully automated NOE system is first briefly described, followed by a systematic examination of possible pilot interfaces with various NOE subsystems. Finally, descriptions of three candidate interface configurations are presented. The configurations were chosen on the basis of the potential for markedly reducing pilot workload and the potential for practical near-term application.

\section{Description of Automated NOE Guidance and Control System (ANGCS)}

Obstacle avoidance (OA) guidance and terrain following in the ANGCS is based upon range measurements assumed to be extracted from either passive or active sensors. Range information is defined according to the resolution of the sensor, and no explicit identification of individual obstacles is assumed. Figure 1 shows a breakdown of the ANGCS (ref. 3). The range information, extracted each cycle, is used to update an inertial database stored as height as a function of horizontal coordinates. The nominal trajectory, depicted in figure 1 as an input to the obstacle-avoidance guidance, is examined for the prediction of a reference point based on the commanded speed of the vehicle. Simultaneously, the inertial database is scanned to discriminate obstacles from the terrain, providing a two-dimensional (2D) range profile as a function of azimuthal look angle in front of the rotorcraft. The $2 \mathrm{D}$ range profile and the predicted reference point are used together to determine a 2D lateral path segment that is free of obstacles. The inertial database is examined over the 2D path segment to provide the altitude profile, resulting in a complete 3D trajectory to be tracked by the autopilot. The current autopilot design employs an aerodynamic inverse model that facilitates the use of a simple linear controller over the whole NOE flight envelope (ref. 7). The output of the autopilot drives the cyclic, collective, and rudder controls directly. 


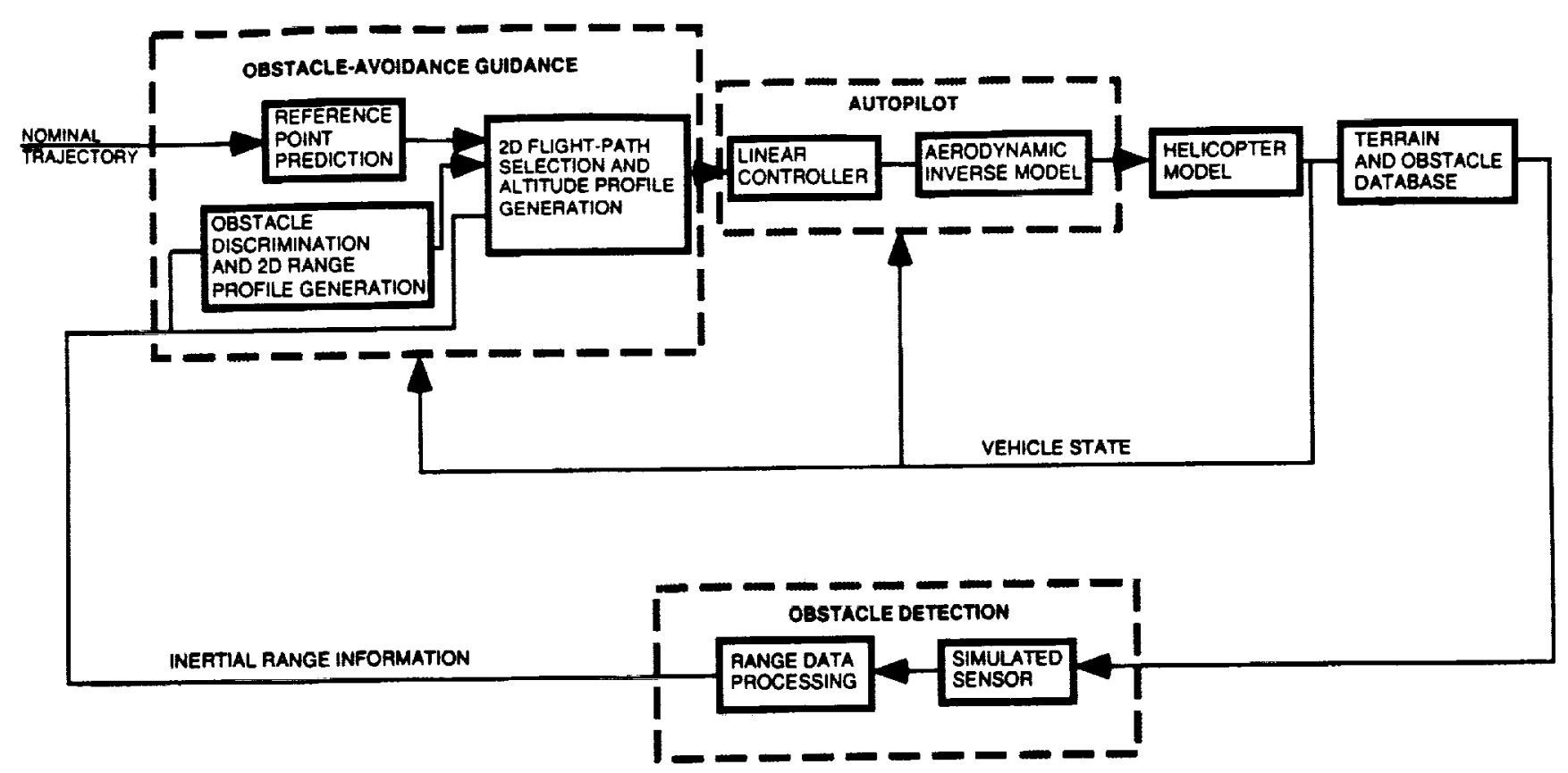

Figure 1. Functional breakdown of automatic NOE guidance and control system (ANGCS).

\section{Configurations of Pilot-Interface Concepts}

The method used in determining possible pilot/ANGCS interaction concepts was simply to categorize both the guidance and the control as being either manual or automatic and to examine the system configurations resulting from the possible combinations. The matrix showing these combinations is given in table 1 . The term "pilot-directed" is used instead of "manual" along the guidance axis because it is more descriptive of the pilot's role, as discussed later. The two guidance categories are further divided according to whether or not a nominal course is to be defined a priori. A primary distinction between automated and pilot-directed guidance is the dependency upon a predefined nominal course. Although not a necessity, human pilots commonly follow nominal predefined courses described by waypoints. On the control axis of table 1 , the manual and automatic control categories are further divided according to whether or not the primary control mode can be overridden. Systems allowing override of primary manual control by the automatic system are considered along with those permitting manual override of primary automatic control. Out of the 16 entries in table 1 , the 7 shaded entries will not be discussed further; reasons are discussed later in this paper. The remaining 9 entries correspond to 9 unique configurations.

The configurations $1 A, B, C$ and $D$ involve the least amount of automation, with the primary guidance and control under the authority of the pilot. Automation in these cases involves the presentation of obstacle position infor- mation, extracted from onboard sensors, to a pilot on a display device, such as a Head-Up Display (HUD) or a Helmet-Mounted Display (HMD), in order to facilitate his own guidance decisions. These configurations are differentiated by whether or not a nominal course is followed and by whether or not control override by an automatic system is allowed. Research in this type of minimal automation for obstacle avoidance is currently ongoing at Ames and elsewhere. Emphasis has been on the presentation of symbology to represent the inertial location of obstacles and the generation of flight-director commands to indicate to the pilot what course of action will eliminate the obstacle threat. Figure 2(a) shows the basic structure of configurations $1 \mathrm{~A}$ and $1 \mathrm{~B}$, which represent manual guidance and control with and without nominal course following, respectively. The system of figure 2(a) would display sensor-derived obstacle database information used in the ANGCS but would leave the formation of guidance commands exclusively to the pilot. Figure 2(b), showing the configurations $1 C$ and $1 D$, is similar to figurc 2(a) cxcept for the addition of automatic inner-loop obstacle-avoidance guidance logic. As in figure 2(a), operation with or without nominal course following is shown. The obstacle-avoidance guidance block in figure 2(b) contains the same data processing and command-generation logic used in ANGCS. In this case, however, the automated reference point would be based on either predicted vehicle location or the pilot's control inputs and not on the nominal trajectory shown in figure 1 . The configuration in figure $2(b)$ corresponds to the case in which the reference point is predicted from the current vehicle statc. Sensor information, 
Table 1. Combinations of manual and automatic guidance and control functions

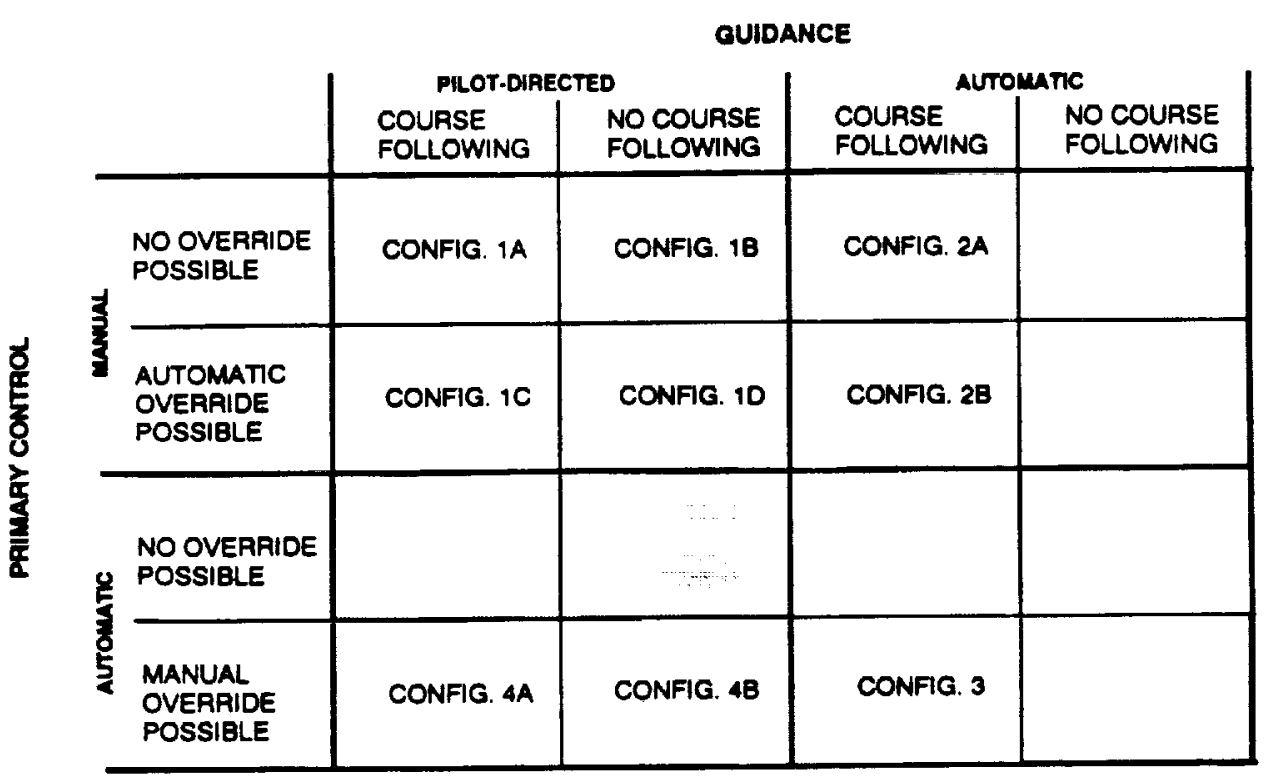

along with vehicle state and trajectory information, is given to the $O A$ guidance, which generates commands to an autopilot in the event that the pilot is approaching an obstacle too closely for safe flight. In this case, the autopilot would override the pilot's controls until the obstacle threat is negotiated.

The system represented by configuration $2 \mathrm{~A}$ is illustrated in figure 3(a). This system displays guidance commands, generated from the ANGCS OA guidance, to be followed by a pilot. In following the commands, the pilot would try to null errors between the current vehicle state and that prescribed by the guidance. Automation based on this type of pilot-system interaction for terrainfollowing terrain-avoidance guidance (TFTA) has already been successfully evaluated in numerous piloted simulations (ref. 8). Configuration 2B, involving an automated "clobber-protection" feature, is shown in figure 3(b). Here, guidance commands are sent to an autopilot in parallel with the pilot's display device in the event that the helicopter appears to be approaching obstacles too closely. As with the concept of figure 2(b), all system components would have to be fail-safe for practical implementation of automatic override of pilot control. It should be pointed out, however, that in any interface system the pilot will have an ultimate means by which to shut down all automation features and resume normal manual control.

The pilot-interface configuration labeled as configuration 3 in table 1 is shown schematically in figure 4 . With
OA guidance commands from the ANGCS provided to the pilot and autopilot in parallel, this concept is similar in structure to that shown in figure 3(b). In this case, however, the autopilot provides the primary control, which can then be modified or overridden by the pilot's control inputs. Without pilot input, OA guidance commands would be given directly to the autopilot, which would generate the control necessary to force the helicopter to follow the commanded trajectory. Trajectory commands from the $O A$ guidance would also be displayed to the pilot (possibly as a flight-path vector/ predictor symbol). By correlating the out-of-window view with the displayed intended trajectory, the pilot can ascertain whether or not any corrective action is necessary to generate a more desirable trajectory. The implementation of figure 4 shows pilot commands (in all axes) being integrated with those from the autopilot to produce a final control vector used to drive the actuators. This scheme, in which pilot commands are combined in some way with the autopilot commands, has been used successfully in many applications including commercial aircraft autopilot systems. If combining inputs is not desirable, pilot inputs in any axis beyond a set threshold (usually a force constraint) can be used to disengage the automatic system and assume fully manual control. This interpretation of pilot input as simply an override constitutes the baseline automatic system, in view of our earlier statement that any interface system would have the override provision. Such a system was used in the evaluation of the STI automated NOE system (ref. 6). 


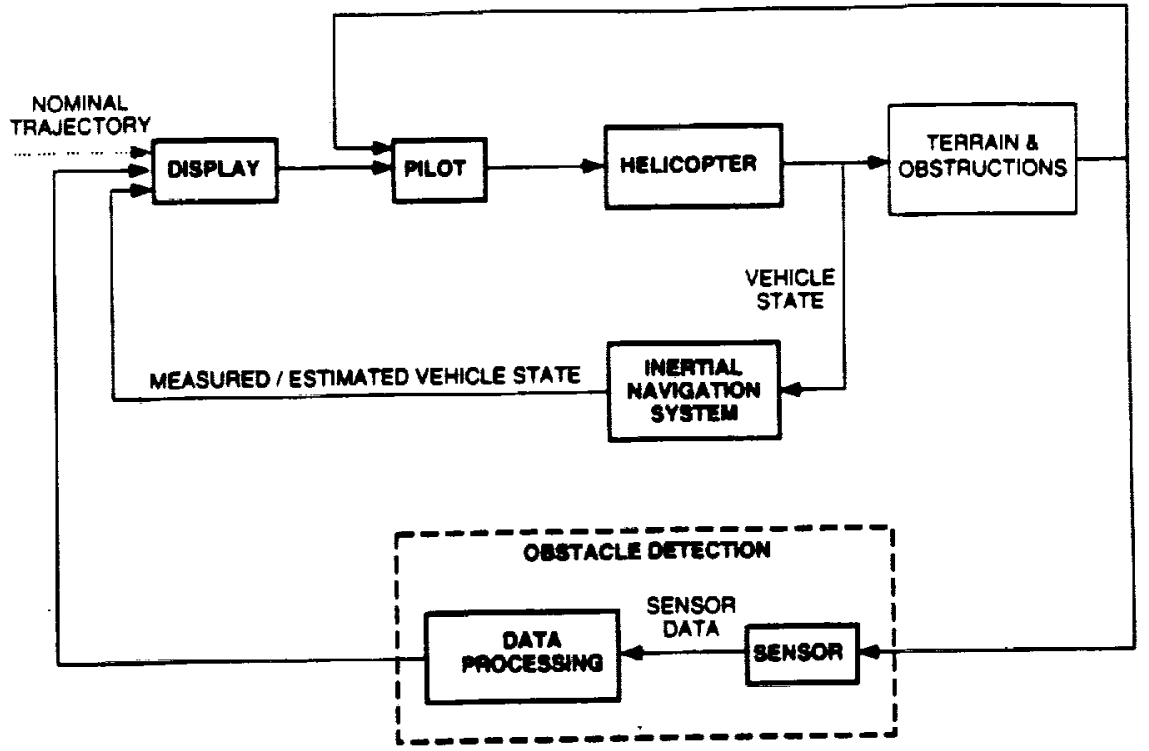

(a) Configurations $1 \mathrm{~A}$ and $1 \mathrm{~B}$.

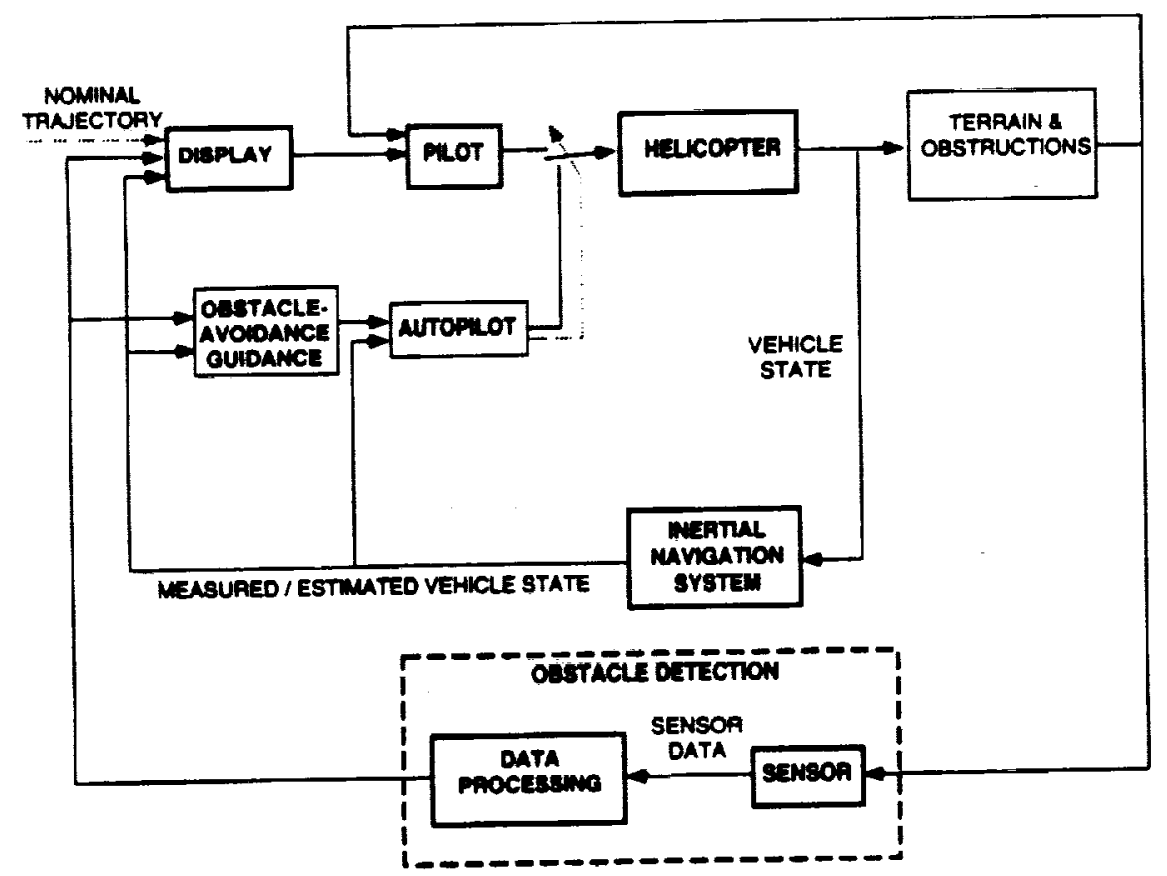

(b) Configurations $1 \mathrm{C}$ and $\mathrm{ID}$.

Figure 2. Configurations $1 \mathrm{~A}, 1 \mathrm{~B}, 1 \mathrm{C}$, and $1 \mathrm{D}$. 


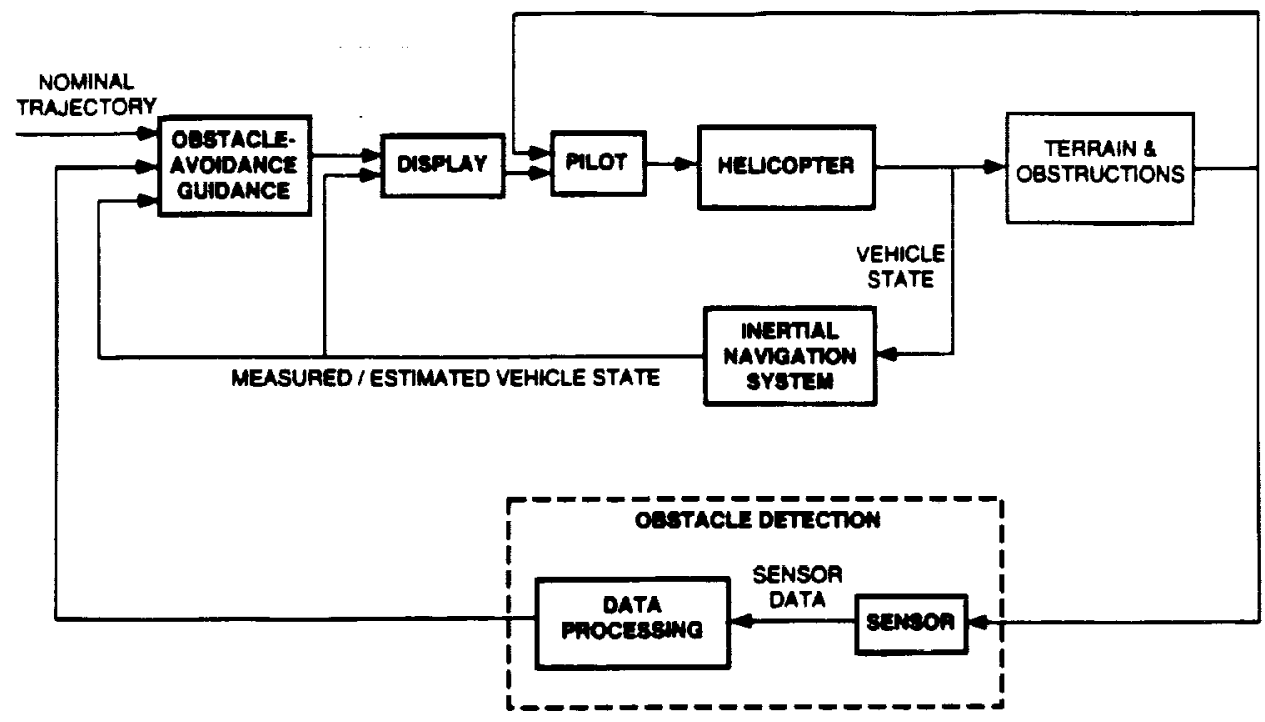

(a) Configuration 2A.

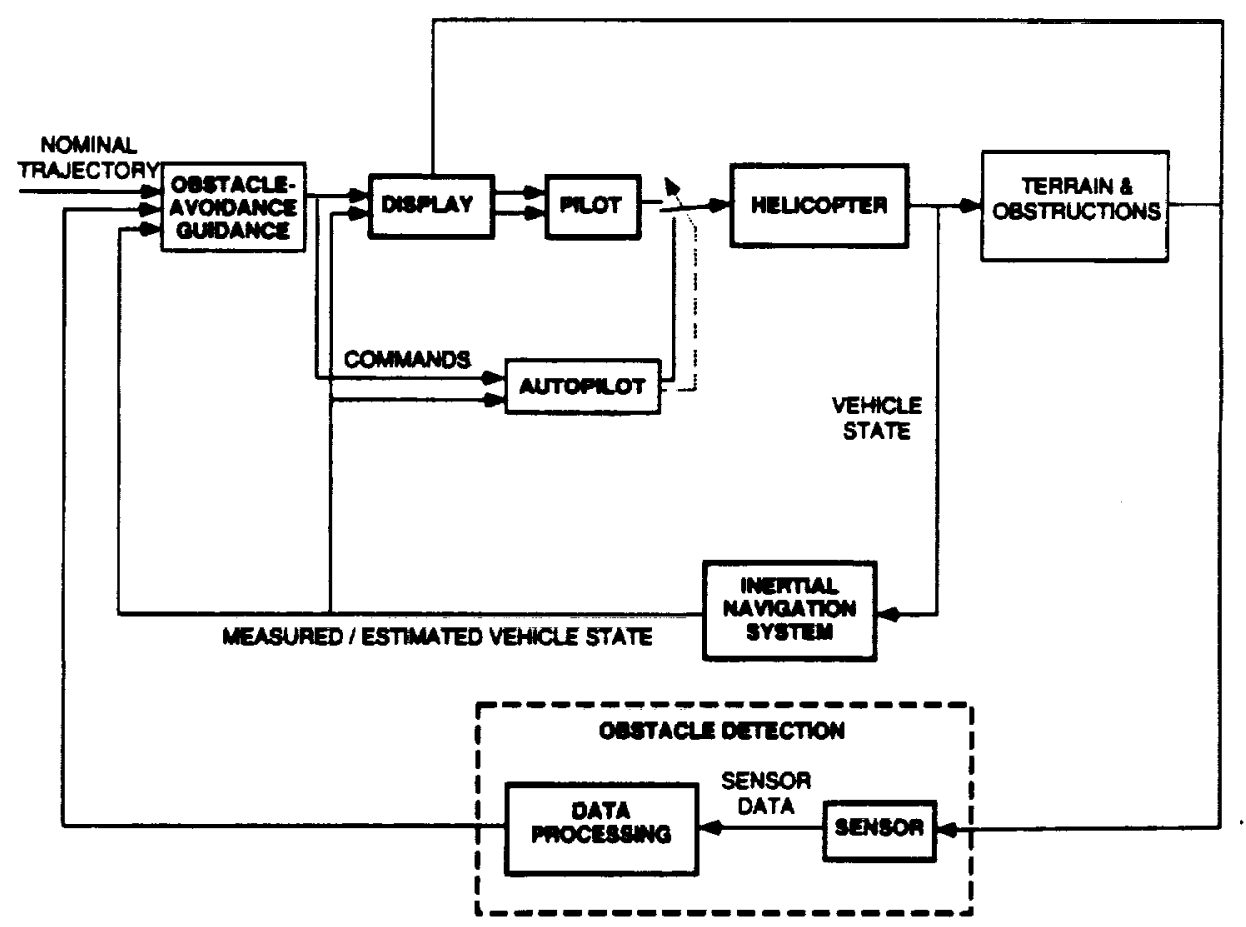

(b) Configuration 2B.

Figurc 3. Configurations $2 \mathrm{~A}$ and 2B. 


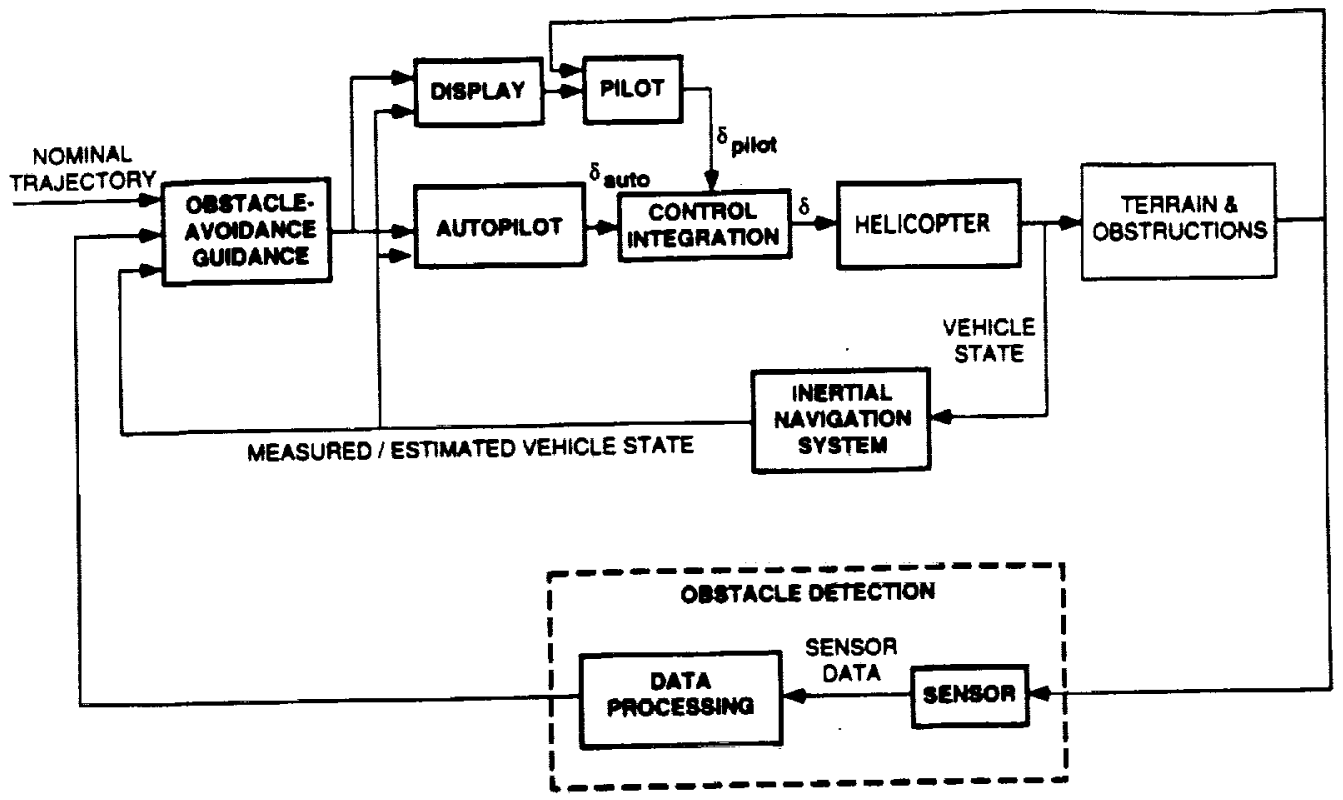

Figure 4. Configuration 3.

Figurc 5 shows a block diagram of the system resulting from manual authority over the guidance and automatic authority over the control, corresponding to configurations $4 A$ and $4 B$ of table 1. Configurations $4 A$ and $4 B$ are differentiated only by the absence of a nominal path reference in 4B. Pilot commands are shown as high-level inputs to the obstaclc-avoidance guidance along with nominal trajectory information (4A only). The final guidance commands are calculated by using these inputs along with obstacle information from the onboard sensor(s). It can be seen that, in the absence of immediate obstacle threats, the final guidance commands to the autopilot will result entircly from the pilot's input and the nominal path, if used. This system is similar in structure to fly-by-wire velocity vector control-whecl-stcering (CWS) systerns where pilot inputs arc interpreted as velocity or trajectory commands to an autopilot (rcf. 9).

\section{Selection of Candidate Interface Concepts}

The shaded entrics of table 1 represent those pilot-system interface concepts that arc cither impractical or not technically feasible. The shaded column on the right side of table 1 represents those systems incorporating automatic navigation and guidance without any type of nominal course following. Thesc systems are considered infeasible since there is currently no practical way for an automatic system to perform purcly autonomous high-level coursc guidance. The remaining shaded blocks represent impractical system configurations in which there are no provisions for pilot override or modification of autopilot control inputs.
Of the nine possible pilot-system interface concepts of table 1 , three were selected for initial testing through piloted simulation. The choice of configurations 3, 4A, and $4 B$ was based upon (1) the potential for significant cockpit workload reduction; (2) the potential for practical implementation; and (3) the usefulness in providing pilot evaluation of the ANGCS technology. All systems involving automatic override of manual control were eliminated from initial consideration because of anticipated problems with pilot acceptance. Configurations $1 \mathrm{~A}$ and $1 \mathrm{~B}$, although recognized as valuable concepts, were not considered for evaluation in this study because they are being adequately investigated elsewhere, and they do not provide a means by which to validate the ANGCS technologies. Configuration 2A was climinated from this initial research since, without careful consolidation of guidance commands, it does not promise significant reductions in pilot workload for NOE flight conditions.

The candidate pilot-system interaction concepts have been given descriptive names for future reference in this paper. The concept described by configuration 3 will be referred to as Pilot-Corrected Control (PCC) since pilot stick and pedal inputs essentially add corrections to the control commands originating from the autopilot. Configuration $4 \mathrm{~A}$ will be referred to as Pilot-Corrected Guidance (PCG) since pilot inputs add corrective inputs to the high-level guidance commands generated from the nominal trajectory. Configuration $4 B$ is referred to as Pilot-Directed Guidance (PDG) since, in the absence of a nominal course, the high-level guidance of the rotorcraft is driven exclusively by pilot stick motions. The location of pilot input to the ANGCS and associated level of automation for each of 


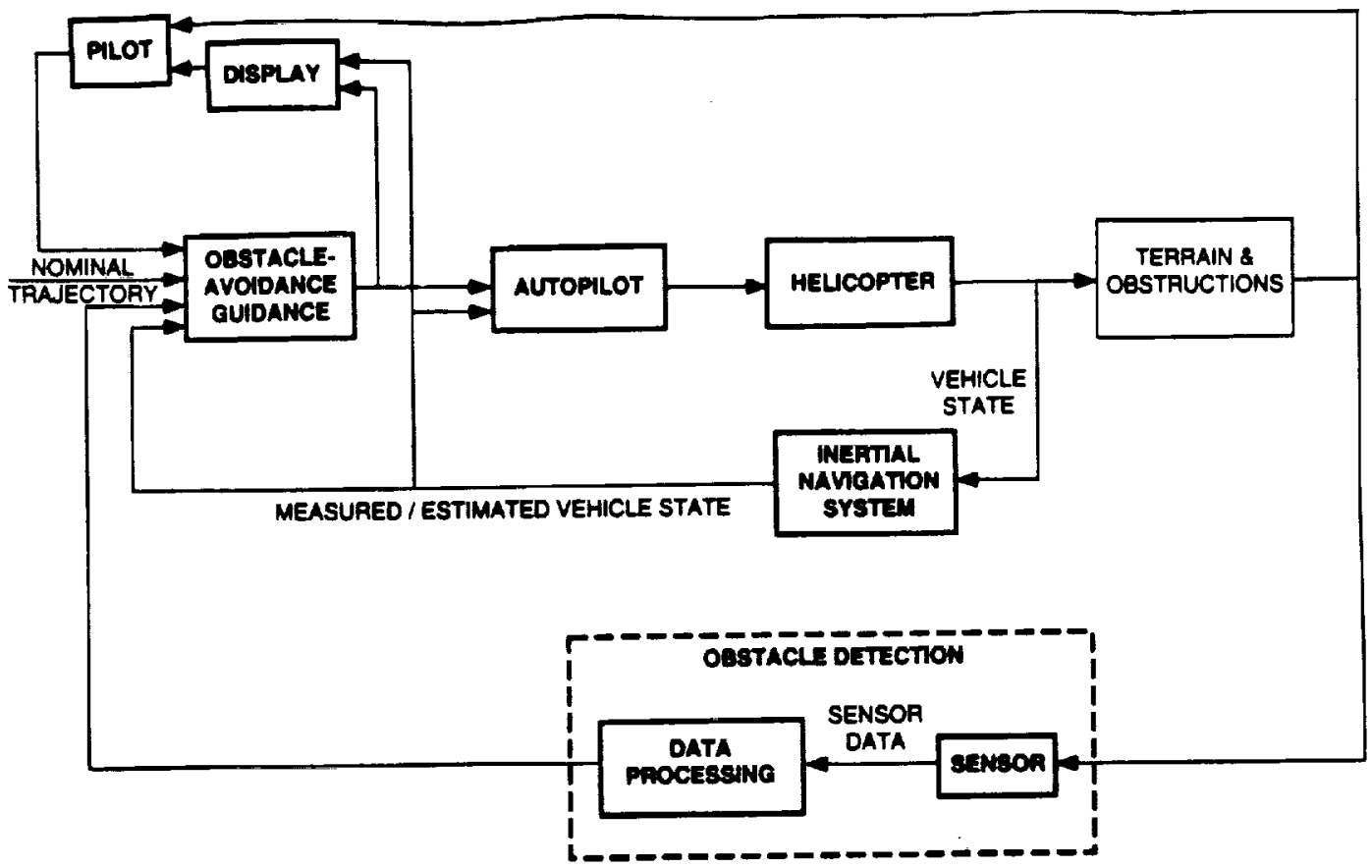

Figure 5. Configurations $4 A$ and $4 B$.

the three candidate interface concepts is shown symbolically in figure 6. The implementation of each candidate concept, developed for integration with the ANGCS, will now be discussed.

\section{Implementation}

\section{Pilot-Corrected Control (PCC)}

As indicated in figure 6, PCC is the candidate concept involving the lowest level of interpretation of pilot control inputs. In PCC, the pilot interacts in a supervisory manner with the ANGCS. In the absence of pilot inputs, the system will behave precisely as the fully automated ANGCS. The simplest implementation of PCC is that shown in figure 7(a), where filtered pilot inputs (cyclic, collective, and pedals) are summed with the inputs computed by the autopilot, forming the total control input to the vehicle. This implementation was withdrawn from consideration, however, because the linearized closedloop system would treat the pilot input as disturbance and proceed to counter it.

The implementation of PCC chosen in this study for pilot evaluation is one in which pilot inputs are combined with automatic inputs within the controller itself. This implementation, shown symbolically in figure 7(b), takes advantage of the structure of the controller and inverse dynamic model that forms the autopilot of the ANGCS. Pilot inputs are now interpreted as velocity commands that modify the velocity commands generated by the $O A$ system. The fundamental difference between this configuration and the one shown in figure $7(a)$ is that feedback of the estimated vehicle state now enters the controller after the summing node, where the pilot input is added to the velocity command generated by the automatic system.

The output from the OA guidance is a horizontal position vector $\Delta r_{H}$ which, relative to the vehicle, indicates the direction of either a reference point on the nominal trajectory ahead of the vehicle or, in the presence of obstacles, an open-path segment. From this inertial position vector, the controller computes a two-component horizontal velocity command as

$$
v_{H_{\text {ouro }}}=v_{d} \frac{\Delta r_{H}}{\left\|\Delta r_{H}\right\|}
$$

where $V_{d}$ is the desired nominal ground speed. The vertical velocity command is calculated from

$$
V_{z_{\text {auso }}}=-V_{d} \tan Y_{d}
$$

where $y_{d}$ is the desired flight-path angle calculated from the terrain altitude profile. The pilot's control inputs are interpreted as additional velocity commands that augment those in equations (1) and (2) generated by the automatic system. For the low NOE flight speeds 


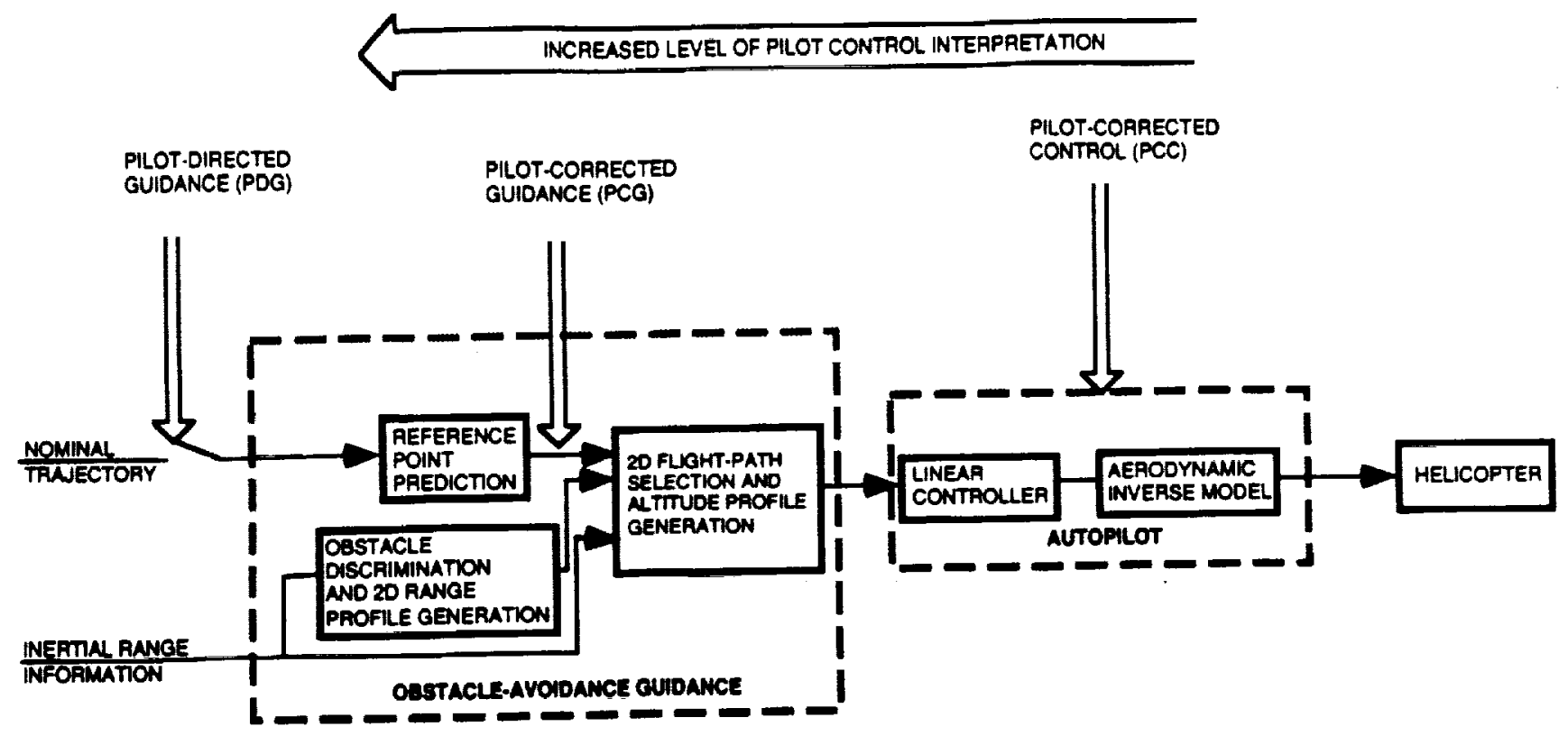

Figure 6. Candidate concepts: points of interface with ANGCS.

$\left(0<V_{d}<40\right.$ knots) considered in this evaluation, pilot control inputs can safely be interpreted as pure translational velocity commands in the vehicle body axes. With the commanded velocity components assumed to be proportional to stick inputs, the resulting inertial velocity command $V_{c}$ is given by

$$
\left[\begin{array}{l}
V_{H x_{\text {pitor }}} \\
V_{H y_{\text {pilen }}} \\
V_{z_{\text {pilet }}}
\end{array}\right]-T_{I B}\left[\begin{array}{ll}
k_{\delta_{\text {ion }}} & \delta_{\text {lon }} \\
k_{\delta_{l o t}} & \delta_{l a t} \\
k_{\delta_{\text {col }}} & \delta_{\text {col }}
\end{array}\right]
$$

where $T_{I B}$ is the body-to-inertial transformation, $\delta$ Ion' $\delta_{l a r}$, and $\delta_{c o l}$ are the pitch cyclic, roll cyclic, and collective inputs, and $k$ represents the corresponding gains. The final horizontal and vertical velocity commands are computed by summing the contribution from the pilot and the OA guidance, i.e.,

$$
\begin{aligned}
& V_{H_{c}}=V_{H_{\text {euto }}}+V_{H_{\text {pilot }}} \\
& V_{z_{c}}=V_{z_{\text {eusus }}}+V_{z_{\text {pilem }}}
\end{aligned}
$$

from which the commanded incrtial acceleration is computed by

$$
\begin{gathered}
a_{H_{c}}=\frac{1}{\tau_{v}}\left(V_{H_{c}}-V_{H}\right) \\
a_{z_{c}}-k_{r}\left(r_{z_{c}}-r_{z}\right)+k_{v}\left(V_{z_{c}}-V_{z}\right)-g
\end{gathered}
$$

where $\tau_{v}, k_{r}$, and $k_{v}$ are control-law constants, $g$ is the acceleration due to gravity, and $V_{H}$ and $V_{z}$ are the current inertial velocity state. As seen in equation (5), vertical acceleration command also depends upon the difference between commanded altitude and estimated altitude $r_{z}$, where commanded altitude is the sum of the terrain altitude below the vehicle and the desired height above ground. The commands in equation (5) are subjected to limits to ensure that they are flyable (ref. 3 ). The modified acceleration commands then form the input to the inverse dynamic model from which the required control positions are calculated.

An important aspect of PCC is the requirement for pilots to know the amount of control authority being used by the autopilot. This requirement can be achieved most directly by back-driving the control sticks and pedals with control commands generated by the autopilot. Furthermore, all pilot inputs should be sufficiently dead-band limited in order to allow the pilot to fly in fully automatic mode while keeping his hands and feet on the controls in anticipation of any emergencies requiring manual-control compensation. This method of supervising automated tasks has proved in the past to be highly acceptable to pilots (ref. 6). 


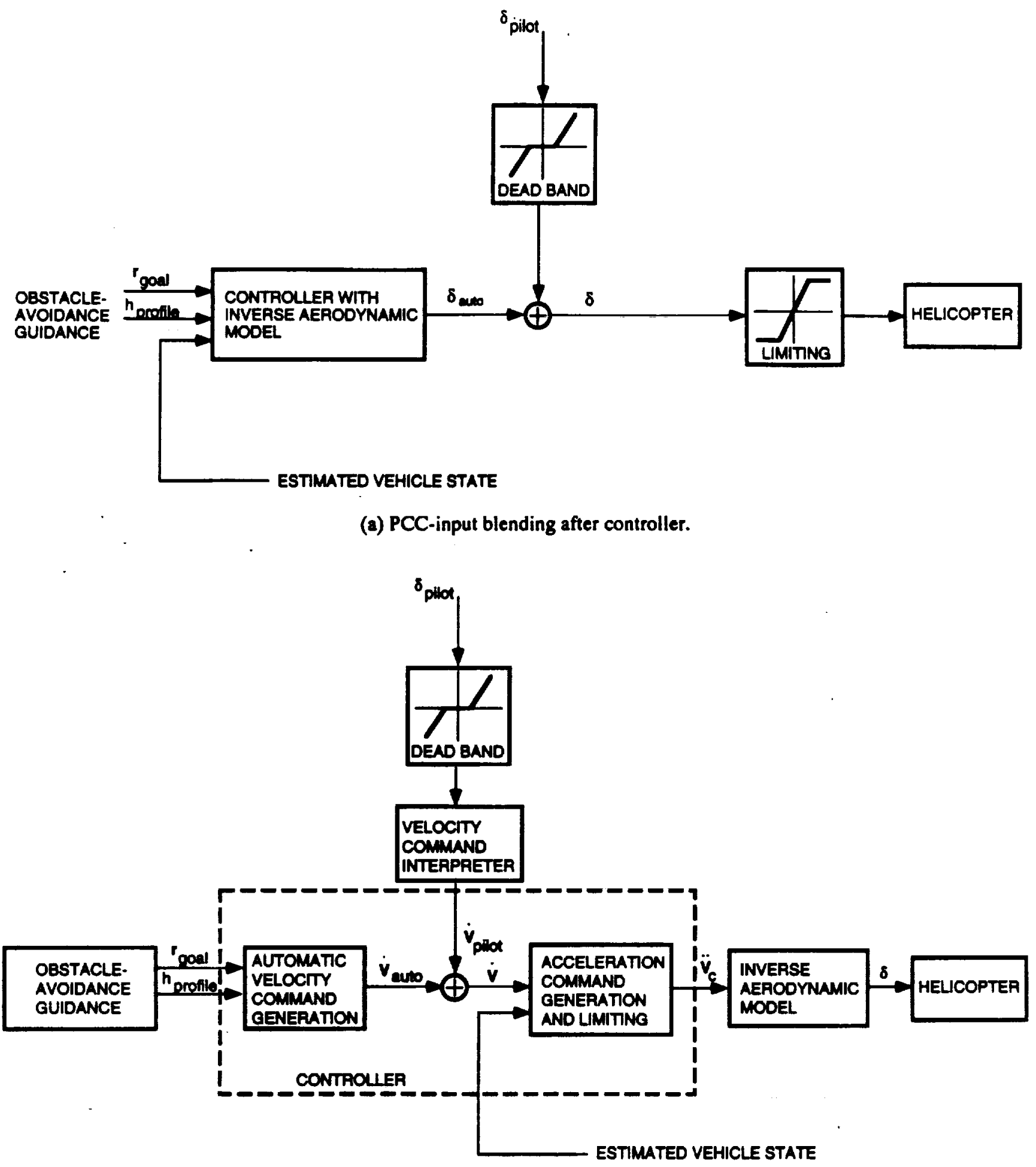

(b) PCC-input blending within controller.

Figure 7. Pilot-corrected control.

Pilot-Corrected Guidance (PCG)

PCG incorporates a higher level of pilot interface than PCC because pilot controls are interpreted as inputs to the automatic guidance system. Since the inputs are interpreted ahead of the automatic OA subsystem, the presence of obstacles may cause the final trajectory command from the autopilot to differ from that intended by 
the pilot. With the assumption of reliable sensors and $O A$ guidance, this system provides a clobber-protection capability not inherent with PCC.

In PCG pilot inputs alter the inertial reference point location, which is calculated ahead of the vehicle on the nominal trajectory. With reference to figure 8 , where visual display of the predicted reference point is assumed but omitted, pilot cyclic inputs are converted to translational velocity commands via equation (3). This velocity command is then used to compute a reference point modification given by

$$
\Delta r_{p i l o t}=\tau_{p} V_{c}
$$

where $\tau_{p}$ is a look-ahead time parameter that could be selectable by the pilot. The final reference point is then given simply as

$$
r_{\text {ref }}=r_{\text {auto }}+\Delta r_{\text {pilot }}
$$

As with PCC, this system will fly under autonomous guidance and control in the absence of pilot input. A similar requirement also exists for back-driving automatic-control inputs and dead-band limiting pilot inputs.

\section{Pilot-Directed Guldance (PDG)}

As with PCG, pilot inputs in the PDG interface are used to control the high-level guidance of the rotorcraft (i.e., prior to modifications by the OA system). In PDG, however, the inertial-reference-point location is influenced by the pilot's controls alone with no dependence upon a nominal trajectory. The PDG interface of the pilot with the ANGCS is described by the block diagram in figure 9. By controlling the reference-point location, the pilot determines the general direction of travel regardless of the presence of obstacles. In the event that a clear path to the reference point is blocked by obstacles, the $O A$ guidance determines an alter- nate path that clears the obstacles, taking into account the physical dimensions of the vehicle. A primary distinction of PDG compared with the other candidate concepts is that the system will not allow the vehicle to fly autonomously in the absence of pilot input.

In PDG, the pilot's control-stick input would define the reference point. Since control of the reference point is twodimensional, only two pilot inputs are required to fix its location. The approach used here is to determine a pseudotrajectory on which the reference point lies based on the orientation of the vehicle's longitudinal axis and the pilot's roll cyclic input $\delta_{\text {lat }}$, used to command lateral acceleration. The reference point is then fixed by determining its arc-length distance along the pseudotrajectory. This distance is controlled by the pilot via a thumb-wheel position $\delta_{t w}$ for commanding prediction time, and pitch cyclic input $\delta$ for commanding ground speed. The effect of the pilot's controls is given by

$$
\begin{aligned}
& \dot{v}_{d}=k_{l o n} \delta_{l o n} \\
& \phi_{c}=\phi+k_{\delta_{l o t}} \delta_{l a t} \\
& \dot{h}_{c l}=k_{\delta_{c o l}} \delta_{c o l} \\
& \tau=k_{\delta_{t w}} \delta_{t w}
\end{aligned}
$$

It should be noted that desired ground speed rate $\dot{V}_{d}$ and commanded terrain clearance rate $\dot{h}_{c l}$ are calculated proportional to pilot pitch cyclic and collective inputs, respectively. Lateral control of the trajectory is accomplished by computing a pseudo bank attitude command $\phi_{c}$, formed by adding a term proportional to the lateral cyclic input to the vehicle's current roll attitude. The distance of the reference point along the pseudotrajectory is computed as the product of the desired speed and a time constant $\tau$, proportional to thumb-wheel position. The commanded attitude corresponds to a constant lateral-acceleration command and

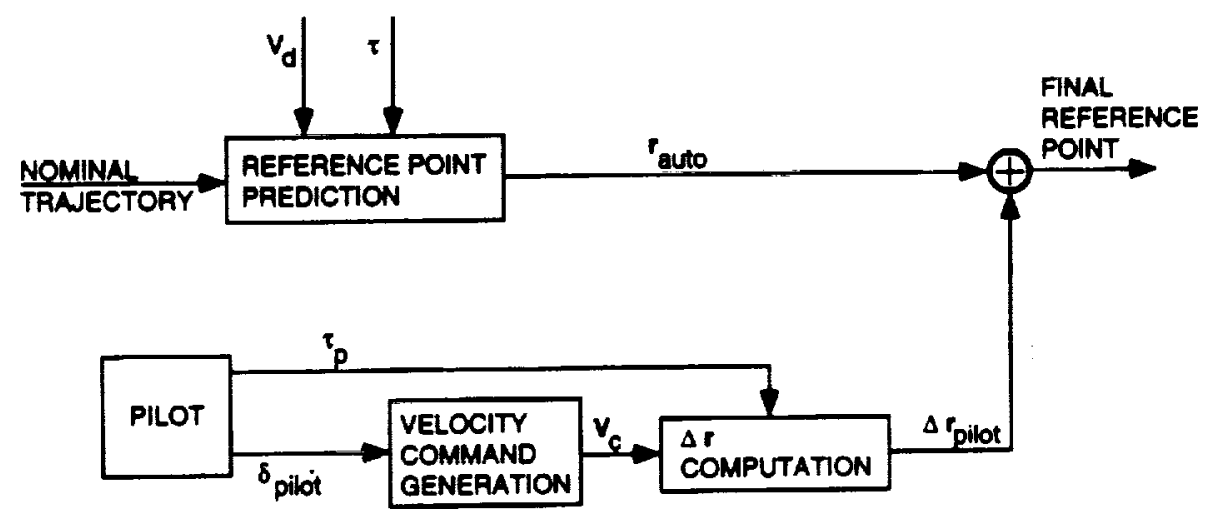

Figure 8. Pilot-corrected guidance implementation. 


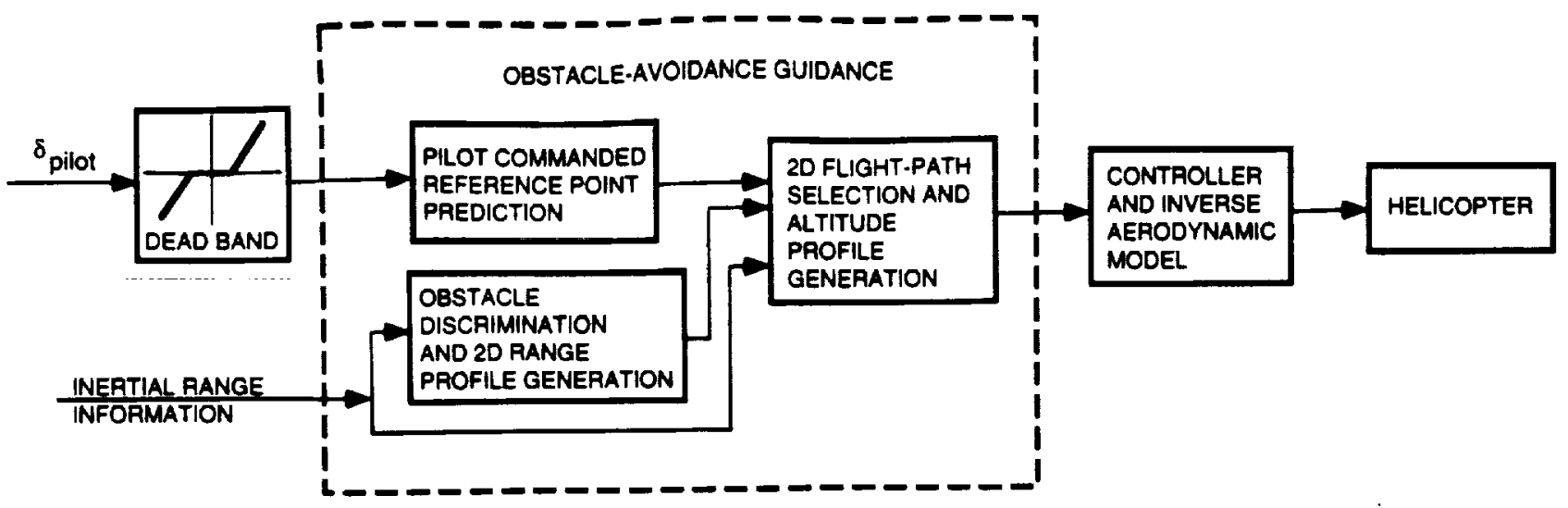

Figure 9. Pilot-directed guidance (PDG).

results in a circular trajectory with a radius of curvature given by

$$
R=\frac{V_{d}^{2}}{g \sin \phi_{c}}
$$

Figure 10 shows the geometry of this reference-point calculation. The inertial location of the reference point is given by

$$
r=\left[\begin{array}{l}
x_{0} \\
y_{0}
\end{array}\right]+\Psi\left[\begin{array}{c}
R \sin \eta \\
R(1-\cos \eta)
\end{array}\right]
$$

where $\Psi$ is the $2 D$ heading cosine matrix for body-to-inertial transformation and $\eta$ is the subtended angle shown in figure 10.

\section{Conclusion}

The problem of pilot interface with an automated NOE rotorcraft system has been addressed via a broad and systematic examination of possible interface concepts and configurations. The criteria of reducing pilot workload and using current technologies were used in selecting three candidate configurations: Pilot-Corrected Control (PCC), Pilot-Corrected Guidance (PCG), and Pilot-Directed Guidance (PDG). Fixed-base piloted simulations to evaluate these concepts are currently under way at Ames, with phase 1 evaluation of PDG completed. Following phase 1 iesting, conclusions will be drawn concerning the utility of each interface concept and the ability of each configuration to reduce pilot workload and improve pilot confidence in automated NOE guidance and control functions. Following pilot-recommended modifications, phase 2 evaluation will involve a series of simulations carried out on the Ames Vertical Motion Simulator.

\section{References}

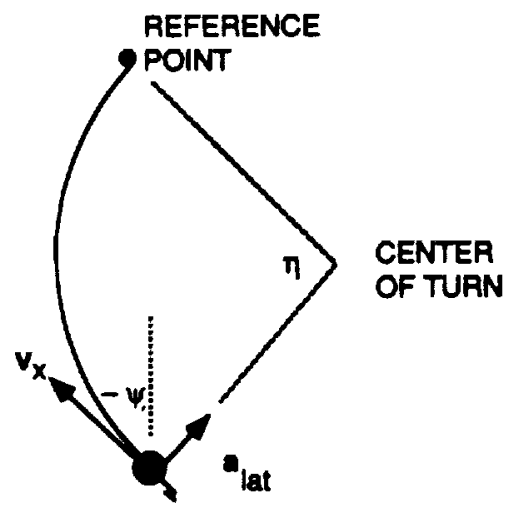

Figurc 10. PDG trajcctory geomctry.
1. Cheng, V. H. L.; and Sridhar, B.: Considerations for Automated Nap-of-the-Earth Flight. AHS Journal, vol. 36, no. 2, April 1991, pp. 61-69.

2. Cheng, V. H. L.: Concept Development of Automatic Guidance for Rotorcraft Obstacle Avoidance. IEEE Transactions on Robotics and Automation, vol. 6, no. 2, April 1990, pp. 252-257.

3. Cheng, V. H. L.; and Lam, T.: Automatic Guidance and Control for Helicopter Obstacle Avoidance. Proceedings of the IEEE International Conference on Robotics and Automation, Nicc, France, May 10-12, 1992, pp. 252-260. 
4. Sridhar, B.; Cheng, V. H. L.; and Phatak, A. V.: Kalman Filter Based Range Estimation for Autonomous Navigation Using Imaging Sensors. Proceedings of the 11 th IFAC Symposium on Automatic Control in Aerospace, Tskuba, Japan, July 1989.

5. Menon, P. K. A.; and Sridhar, B.: Image Based Range Determination. Proceedings of the AIAA Guidance, Navigation, and Control Conference, Portland, OR, August 1990.

6. Clement, W. F.; Gorder P. J.; Jewell, W. F.; and Coppenbarger, R. A.: Real-Time Piloted Simulation of Fully Automatic Guidance and Control for Rotorcraft Nap-of-the-Earth (NOE) Flight Following Planned Profiles. Proceedings of the AlAA Guidance, Navigation, and Control Conference, Portland, OR, August 1990.
7. Heiges, M. W.: A Helicopter Flight Path Controller Design via a Nonlinear Transformation Technique. Ph.D. Dissertation, Georgia Institute of Technology, March 1989.

8. Swenson, H.: Computer Aiding for Low-Altitude Helicopter Flight. AHS Annual Forum, Phoenix, AZ, May 6-8, 1991.

9. Wehrend, W. R., Jr.: Pilot Control Through the TAFCOS Automatic Flight Control System. NASA TM-81152, 1979.

10. Lam, T.; and Cheng, V. H. L.: Simulation of Automatic Nap-of-the-Earth in Graphics Workstation Environment. Proceedings of the AIAA/AHS Flight Simulation Technologies Conference, August 24-26, 1992. 



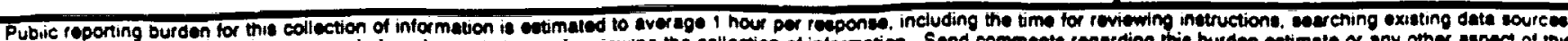
athering and maintaining the date needed, and completing end reviowing the collection of imbormation. Send comments iegerding this burden eatimale or any other aspect of this

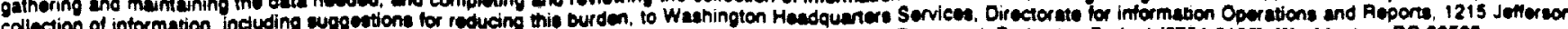
Devis Highway, Suite 1204 , Allington. VA 20202-4302, and to the Othce of Management and Budget. Paperwork Aeduction Project (0704-01Be). Waehington. DC 20503.

\begin{tabular}{l|l} 
1. AGENCY USE ONLY (Leave blank) & 2. AEPOAT DATE
\end{tabular}

December 1992

3. REPOAT TYPE AND DATES COVERED

Technical Memorandum

4. TITLE AND SUBTITLE

Concepts for Pilot Interaction with an Automated NOE Obstacle-

Avoidance System

6. AUTHOR(S)

$505-64-36$

R. A. Coppenbarger and V. H. L. Cheng

7. PERforming ofganization mame(S) AND adofess(ES)

- Performino organization REPORT NUMBER

Ames Research Center

Moffett Field, CA 94035-1000

A-92157

9. SPONSOAING/MONITOAING AOENCY MAME(S) ANO ADORESS(ES)

10. SPONSORING/MONITORINO

AGENCY REPORT NUMBEA

National Aeronautics and Space Administration

Washington, DC 20\$̣46-0001

NASA TM-103960

\section{SUPPLEMENTARY NOTES}

Point of Contact: Richard Coppenbarger, Ames Research Center, MS 210-9, Moffett Field, CA 94035-1000; (415) $604-5433$

Unclassified - Unlimited

Subject Category 08

\section{ABSTRACT (Maximum 200 words)}

The problem of providing an appropriate method by which a human pilot interacts with an automated nap-ofthe-Earth rotorcraft guidance and control system is addressed. This problem is closely related to the broader question of what level and degree of automation is effective at reducing pilot workload during low-altitude flight missions requiring obstacle avoidance. A systematic approach for establishing the possible combinations of manual versus automatic authority over relevant guidance and control functions is first presented. From these possibilities, three candidate concepts are selected; selection is based upon their potential for practical implementation and reduction in pilot workload. This paper describes the selection of these three pilot-interaction concepts and the mathematical models for their implementation.

\section{SUBJECT TEAMS}

Guidance, Nap-of-the-Earth, Pilot interface, Obstacle-avoidance

17. SECUAITY CLASSIFICATION

OF AEPOAT

Unclassified
19. SECUAITY CLASSIFICATION OF ABSTRACT

SECUAITY CLASSIFICATIOH
OF THIS PAOE
Unclassified

Unclassified
20

20. LIMITATION OF ABSTAACT

Standard Form 298 (Rov. 2-89) 